\title{
On the vanishing of higher syzygies of curves
}

\section{Marian Aprodu}

Romanian Academy, Institute of Mathematics "Simion Stoilow", P.O.Box 1-764, RO70700, Bucharest, Romania (e-mail: Marian.Aprodu@imar.ro) \&

Université de Grenoble 1, Laboratoire de Mathématiques, Institut Fourier BP 74, 38402

Saint Martin d'Hères Cedex, France (e-mail: aprodu@mozart.ujf-grenoble.fr)

\section{Introduction, main results}

A main challenge in the theory of syzygies is to interpret the information carried by the graded Betti numbers of a smooth projective variety. Notably, the attempt to understand the way in which the distribution of zeroes in a Betti table interacts with the geometry of the variety has led to a considerable amount of work, motivated by the conjectures that Green, Green-Lazarsfeld, and others had formulated (see, for example, [Gr1], GL1], EL, [La2]).

One of the most significant conjectures made by Green and Lazarsfeld (cf. GL1] 3.7; see also Gr3] 3.5 and [La2 2.3), nowadays known as the gonality conjecture, predicts that one could read off the gonality of a smooth complex projective curve from the minimal resolution of any line bundle of sufficiently large degree. In order to give a precise statement, the authors introduced the vanishing property $\left(M_{k}\right)$ (see [GL1], Gr3]), which is the following (we use the notation of Section 1).

Definition. (Green-Lazarsfeld) If $X$ is a smooth complex projective curve of genus $g, L$ a line bundle on $X$, and $k \geq 0$ an integer, one says that $(X, L)$ satisfies the property $\left(M_{k}\right)$ (or, simply, $L$ satisfies $\left(M_{k}\right)$ ) if $K_{p, 1}(X, L)=0$, for all $p \geq h^{0} L-k-1$.

It is well-known (see, for example, GL1, GL2 or Sch1) that if $X$ carries a $g_{k}^{1}$, then no line bundle of sufficiently large degree can satisfy $\left(M_{k}\right)$. The gonality conjecture states a converse of this fact: if $\operatorname{deg}(L)>>2 g$, and $\left(M_{k}\right)$ fails for $L$, then $X$ carries a $g_{q}^{1}$, with $q \leq k$. Green has shown this conjecture holds for $k=1,2$ (cf. [Gr1]); Ehbauer proved it in the case $k=3$ (cf. [Ehb]). Thus hyperelliptic and trigonal curves are characterized by syzygies.

The purpose of the present work is to prove that, under certain conditions, the property $\left(M_{k}\right)$ is preserved when we add an effective divisor to a line bundle. The first result is:

Theorem 1. Let $X$ be a smooth complex projective curve of genus $g \geq 1, L_{0}$ be a nonspecial globally generated line bundle on $X$, and $k \geq 0$ be an integer such that the pair $\left(X, L_{0}\right)$ satisfies the property $\left(M_{k}\right)$. Then, for any effective divisor $D$ on $X$, the pair $\left(X, L_{0}+D\right)$ satisfies the property $\left(M_{k}\right)$. In particular, for any line bundle $L$ with $\operatorname{deg}(L) \geq \operatorname{deg}\left(L_{0}\right)+g$, the pair $(X, L)$ satisfies the property $\left(M_{k}\right)$ as well.

There are a number of immediate consequences of Theorem 1. For instance, if $X$ carries a $g_{k}^{1}$, and $L_{0} \in \operatorname{Pic}(X)$ is nonspecial, and globally generated, then $\left(X, L_{0}\right)$ cannot satisfy $\left(M_{k}\right)$. Further, the least $k$ for which the property $\left(M_{k}\right)$ fails for a 
nonspecial, globally generated line bundle cannot decrease when adding effective divisors, and cannot pass over the gonality of $X$, and thus it must be constant when the degree of the line bundle grows large enough. The challenge of the gonality conjecture is to show that this constant always equals the gonality of $X$.

In view of Theorem 1 , verifying the gonality conjecture for a given $k$-gonal $X$ of genus $g$ reduces to finding a single nonspecial, globally generated line bundle (for example, a line bundle whose degree is sufficiently large compared to $2 g$ ), with property $\left(M_{k-1}\right)$. Concretely, we get the following criterion for testing the gonality conjecture:

Corollary 2. Let $X$ be a smooth complex projective curve of genus $g \geq 1$, which carries a $g_{k}^{1}$, and $L_{0}$ be a nonspecial, globally generated line bundle on $X$ satisfying the property $\left(M_{k-1}\right)$. Then $X$ is $k$-gonal, and the gonality conjecture is valid for $X$.

By semicontinuity of minimal resolutions, and irreducibility of the moduli space $\mathcal{M}_{g, k}$ of $k$-gonal curves of genus $g$ (cf. $[\mathbb{F}]$ ), it turns out that the gonality conjecture would also be valid for a generic curve in $\mathcal{M}_{g, k}$, once it was verified for a particular $k$-gonal curve of genus $g$. It seems thus very reasonable to predict that for any positive integers $k$, and $g$, such that $\mathcal{M}_{g, k} \neq \emptyset$, a generic $k$-gonal curve of genus $g$ verifies the gonality conjecture (see below).

One could address now the question of what happens if we drop the nonspeciality condition in Theorem 1. A partial answer is given by:

Theorem 3. Let $X$ be a smooth complex projective curve of genus $g \geq 1$, and $k \geq 0$ be an integer such that the pair $\left(X, K_{X}\right)$ satisfies the property $\left(M_{k}\right)$. Then, for any effective divisor $D$ on $X$, the pair $\left(X, K_{X}+D\right)$ satisfies the property $\left(M_{k}\right)$.

Unfortunately, the result just stated is weaker than it might look like at a first sight - it does not show that Green's generic canonical conjecture implies the gonality conjecture for a generic curve, as one could think of. The following better version of it would do the job instead.

Conjecture. Let $X$ be a smooth complex projective curve of Clifford dimension one, and $p$ an integer such that $K_{p, 1}\left(X, K_{X}\right)=0$. Then there exist a positive integer $d$, and an effective divisor $D$ on $X$, of degree $d$, such that $K_{p+d-1,1}\left(X, K_{X}+D\right)=0$.

This conjecture is obviously false if we drop the condition that the Clifford dimension be equal to one, as seen by analyzing the case of smooth plane curves. In exchange, it holds for other curves which are not plane curves, such as curves lying on Hirzebruch surfaces (see Sections 6 and 8), and, more generally, it is true for curves which verify both the gonality conjecture, and Green's canonical conjecture (trigonal curves, for instance).

The main ingredient we use to prove Theorem 1, and Theorem 3, is projection of syzygies, concept which was introduced by Ehbauer [Ehb], in a coordinate-based manner. In Section 2, we propose a more abstract view on the subject, in the spirit of Gr2 1.b.1, and we think of projections of syzygies as being corestrictions of 
the fiber-restrictions of a certain morphism between vector bundles. We investigate some properties of the projection morphisms, and we show, among other things, that, under some conditions which are almost always satisfied, any nonzero syzygy survives by projection from a generic point (see (2.5)).

The third Section deals with projection of syzygies of varieties. We recall here Ehbauer's approach, and examine the case of syzygies of curves.

In Section 4 we complete the proofs of Theorem 1, and Theorem 3, and further, in the final part of the paper, we analyze some very concrete cases.

We verify first the gonality conjecture for smooth plane curves, and for smooth curves lying on a Hirzebruch surface, in which cases we can make use of the geometry of the ambient surface to produce suitable line bundles. Additionally, we recover the description of the minimal pencils in these cases, which has already been known before (for plane curve, see, for example [ACGH], for curves on a Hirzebruch surface we refer to Ma1]).

In the fifth Section, we test the gonality conjecture for nodal curves on $\mathbf{P}^{1} \times \mathbf{P}^{1}$, case which eventually shows the following (compare with the main result of [Sch2]):

Theorem 4. For any integer $k \geq 3$, the gonality conjecture is valid for a generic $k$-gonal curve of genus $g>(k-1)(k-2)$.

We conclude this paper by applying the vanishing result (6.2) proved here to show that Green's canonical conjecture holds for smooth curves on Hirzebruch surfaces (compare with [LO]). As a general philosophy, we expect that smart choices of line bundles on a surface, whose restrictions satisfy the vanishing property required in the gonality conjecture for some curves lying on that surface, be used to prove Green's canonical conjecture for such curves.

\section{Some notation, preliminaries}

For many of the theoretical facts included in this section we refer to the papers Gr1], Gr3], La1, without further mention. We start with $V$ a finite-dimensional complex vector space, we denote $S V$ the symmetric algebra of $V$, and we consider $B=\bigoplus_{q \in \mathbf{Z}} B_{q}$ a graded $S V$-module. Then there is a naturally defined complex of vector spaces, called the Koszul complex of $B$,

$$
\ldots \longrightarrow B_{q-1} \otimes \bigwedge^{p+1} V \stackrel{d_{p+1, q-1}}{\longrightarrow} B_{q} \otimes \bigwedge^{p} V \stackrel{d_{p, q}}{\longrightarrow} B_{q+1} \otimes \bigwedge^{p-1} V \longrightarrow \ldots
$$

whose cohomology is denoted by

$$
K_{p, q}(B, V)=\operatorname{Ker} d_{p, q} / \operatorname{Im} d_{p+1, q-1} .
$$

The dimensions of the Koszul cohomology spaces are called graded Betti numbers of $B$, and their elements are called syzygies.

An important property of Koszul cohomology is its functorial behavior - any morphism of graded $S V$-modules, $A \rightarrow B$, canonically induces linear maps $K_{p, q}(A, V) \rightarrow$ 
$K_{p, q}(B, V)$. Moreover, to any short exact sequence of graded $S V$-modules, $0 \rightarrow A \rightarrow$ $B \rightarrow C \rightarrow 0$ one associates a long exact sequence, for any $p$ (cf. [Gr1], 1.d.4):

$$
\ldots \rightarrow K_{p+1,0}(C, V) \rightarrow K_{p, 1}(A, V) \rightarrow K_{p, 1}(B, V) \rightarrow K_{p, 1}(C, V) \rightarrow K_{p-1,2}(A, V) \rightarrow \ldots
$$

(1.1) Remark. With the notation above, we see that if $C_{0}=0$, then the map $K_{p, 1}(A, V) \rightarrow K_{p, 1}(B, V)$ is injective, and if $C_{0}$, and $C_{1}$ both vanish, then $K_{p, 1}(A, V) \cong K_{p, 1}(B, V)$.

Notation. If $X$ is an irreducible complex projective variety, $L \in \operatorname{Pic}(X)$ is a line bundle, $\mathcal{F}$ is a coherent sheaf, and $V \subset H^{0}(X, L)$, take $B=\bigoplus_{q \in \mathbf{Z}} H^{0}(X, \mathcal{F} \otimes q L)$ (here, $q L$ is the $q$-th tensor power of $L$ in $\operatorname{Pic}(X))$, and denote $K_{p, q}(X, \mathcal{F}, L, V)=$ $K_{p, q}(B, V)$. If $V=H^{0}(X, L)$, we drop $V$ and write $K_{p, q}(X, \mathcal{F}, L)$, if $\mathcal{F} \cong \mathcal{O}_{X}$, we suppress it, and write $K_{p, q}(X, L, V)$; the notation $K_{p, q}(X, L)$ corresponds to the choice $V=H^{0}(X, L)$ and $\mathcal{F} \cong \mathcal{O}_{X}$.

(1.2) Remark. If $L$ is a globally generated line bundle over the smooth irreducible variety $X$, and $V=H^{0}(X, L)$, by denoting $X^{\prime}$ the image of $X$ in $\mathbf{P} V^{*}$, through the morphism given by the complete linear system $|L|$, and $S_{X^{\prime}}=\operatorname{Im}(S V \rightarrow$ $\left.\oplus H^{0}(X, q L)\right)$ its homogeneous coordinate ring, then, in virtue of (1.1), we have natural isomorphisms $K_{p, 1}\left(S_{X^{\prime}}, V\right) \cong K_{p, 1}(X, L)$, for all integers $p$.

(1.3) Remark. If $X$ is a smooth irreducible projective variety with $h^{1} \mathcal{O}_{X}=0$, $L \in \operatorname{Pic}(X)$ a line bundle, and $Y \in|L|$ is irreducible, then $K_{p, 1}(X, L) \cong K_{p, 1}\left(Y,\left.L\right|_{Y}\right)$ for any integer $p$. For the proof, we apply (1.1), and argue as in 3.b.7 of [Gr1].

Convention. Unless otherwise stated, a curve will always mean a smooth, connected, complex, projective curve.

\section{Projections of syzygies at large}

We consider, as in the previous section, $V$ a finite-dimensional complex vector space, and $B$ a graded $S V$-module. We denote by $\mathbf{P}=\mathbf{P} V^{*}=\operatorname{Proj}(S V)$, and $L=\mathcal{O}_{\mathbf{P}}(1)$. A point $x \in \mathbf{P}$, which corresponds to a short exact sequence of vector spaces:

$$
0 \longrightarrow W_{x} \longrightarrow V \stackrel{\mathrm{ev}_{x}}{\longrightarrow} L_{x} \longrightarrow 0,
$$

naturally induces, for any integer $l$, a short exact sequence of Koszul complexes:

$$
0 \longrightarrow B_{*} \otimes \bigwedge^{l+1-*} W_{x} \longrightarrow B_{*} \otimes \bigwedge^{l+1-*} V \longrightarrow L_{x} \otimes B_{*} \otimes \bigwedge^{l-*} W_{x} \longrightarrow 0
$$

which gives rise to a long exact sequence (compare with Gr2 1.b.1):

$$
(*) \ldots \rightarrow K_{p+1, q}\left(B, W_{x}\right) \stackrel{\eta_{x}}{\rightarrow} K_{p+1, q}(B, V) \stackrel{\pi_{x}}{\rightarrow} L_{x} \otimes K_{p, q}\left(B, W_{x}\right) \stackrel{\mu_{x}}{\rightarrow} K_{p, q+1}\left(B, W_{x}\right) \rightarrow \ldots,
$$

where $p+q=l$. We call the map $K_{p+1, q}(B, V) \stackrel{\pi_{x}}{\longrightarrow} L_{x} \otimes K_{p, q}\left(B, W_{x}\right)$ projection of syzygies centered in $x$, and the elements of its image, projected syzygies. 
Projection of syzygies is functorial, that is, for any morphism $B \rightarrow C$ of graded $S V$-modules, the induced morphisms between syzygies $K_{p+1, q}(B, V) \rightarrow K_{p+1, q}(C, V)$, and $L_{x} \otimes K_{p, q}\left(B, W_{x}\right) \rightarrow L_{x} \otimes K_{p, q}\left(C, W_{x}\right)$ commute with the corresponding projection morphisms. Furthermore, projection of syzygies is compatible with the connecting morphisms arising from the long cohomology sequences: if $0 \rightarrow A \rightarrow B \rightarrow$ $C \rightarrow 0$ is an exact sequence of graded $S V$-modules, then the connecting morphisms $K_{p+1, q}(C, V) \rightarrow K_{p, q+1}(A, V)$, and $L_{x} \otimes K_{p, q}\left(C, W_{x}\right) \rightarrow L_{x} \otimes K_{p-1, q+1}\left(A, W_{x}\right)$ commute with projections.

(2.1) Remark. The composed map $\partial_{x}:=\left(\eta_{x} \otimes \mathrm{id}_{L_{x}}\right) \circ \pi_{x}: K_{p+1, q}(B, V) \rightarrow$ $L_{x} \otimes K_{p, q}(B, V)$ is the fiber-restriction over $x$ of a natural sheaf morphism between two vector bundles:

$$
\mathcal{O}_{\mathbf{P}} \otimes K_{p+1, q}(B, V) \stackrel{\partial}{\longrightarrow} \mathcal{O}_{\mathbf{P}}(1) \otimes K_{p, q}(B, V) .
$$

In order to prove this, we use the Koszul complex of $B$, and suitable shifts of the well-known exact complex of vector bundles on $\mathbf{P}$ :

$$
0 \rightarrow \mathcal{O}_{\mathbf{P}}(-r-1) \otimes_{\mathbf{C}} \bigwedge^{r+1} V \rightarrow \mathcal{O}_{\mathbf{P}}(-r) \otimes_{\mathbf{C}} \bigwedge^{r} V \rightarrow \ldots \rightarrow \mathcal{O}_{\mathbf{P}}(-1) \otimes_{\mathbf{C}} V \rightarrow \mathcal{O}_{\mathbf{P}} \rightarrow 0
$$

to obtain, for each integer $l \in \mathbf{Z}$, a double complex of vector bundles over $\mathbf{P}$ with general term

$$
\mathcal{K}^{s, q}=\mathcal{O}_{\mathbf{P}}(s) \otimes B_{q} \otimes \bigwedge^{l-s-q} V .
$$

Since the rows of this double complex are exact, it yields a spectral sequence abutting to zero, with general term

$$
\mathcal{E}_{1}^{s, q}=\mathcal{O}_{\mathbf{P}}(s) \otimes K_{l-s-q, q}(B, V) .
$$

The differential $\mathcal{O}_{\mathbf{P}} \otimes K_{p+1, q}(B, V) \stackrel{\partial}{\longrightarrow} \mathcal{O}_{\mathbf{P}}(1) \otimes K_{p, q}(B, V)$, obtained at the first level of the above spectral sequence by setting $s=0$ and $p=l-q-1$, is the morphism we were looking for.

In the real life, we often meet graded $S V$-modules which satisfy the following property, which will be assumed to be satisfied for the rest of the Section,

$$
B_{q}=0 \text { for all } q<0 \text {, and } K_{p, 0}(B, V)=0 \text { for all } p \geq 1 \text {. }
$$

Under these assumptions, we are able to prove some properties of the projection of syzygies for $q=1$, as follows.

(2.2) Lemma. The map $K_{p+1,1}(B, V) \stackrel{H^{0}(\partial)}{\longrightarrow} V \otimes K_{p, 1}(B, V)$ is injective.

Proof. We apply $H^{0}$ to $\mathcal{K}^{s, q}$. The new double complex, with general term $H^{0} \mathcal{K}^{s, q}$, gives rise to a spectral sequence such that $E_{1}^{s, q} \cong H^{0} \mathcal{E}_{1}^{s, q}$, and $E_{\infty}^{s, q}=0$ for any pair $(s, q)$, except for $(s, q)=(0, l)$ when $E_{\infty}^{0, l}=B_{l}$. Moreover, $H^{0}(\partial)$ coincides to the differential $E_{1}^{0,1} \rightarrow E_{1}^{1,1}$. Because of the assumptions we have made on $B$, we get $E_{2}^{0,1}=E_{3}^{0,1}=\ldots=E_{\infty}^{0,1}=0$. In particular, $\operatorname{Ker} H^{0}(\partial)=0$. 
(2.3) Lemma. The map $K_{p+1,1}\left(B, W_{x}\right) \stackrel{\eta_{x}}{\longrightarrow} K_{p+1,1}(B, V)$ is injective.

Proof. Use the short exact sequence $(*)$ twice.

(2.4) Remark. The projection morphism $\pi_{x}$ is in this case a genuine corestriction of $\partial_{x}$. Moreover, the following relation also holds: $\partial_{x}=\left(\operatorname{ev}_{x} \otimes \operatorname{id}_{K_{p, 1}(B, V)}\right) \circ H^{0}(\partial)$.

(2.5) Lemma. Any nonzero element $\alpha \in K_{p+1,1}(B, V)$ survives when we project it from a point $x$ outside a projective subspace of $\mathbf{P}$.

Proof. For any nonzero element $\alpha \in K_{p+1,1}(B, V)$, writing $H^{0}(\partial)(\alpha)=\sum v_{i} \otimes \alpha_{i}$, with $\alpha_{i} \in K_{p, 1}(B, V)$ linearly independent and $v_{i} \in V$, we see that $\partial_{x}(\alpha) \neq 0$ as long as the point $x$ does not belong to the projective subspace of $\mathbf{P},\left\{y \in \mathbf{P}, v_{i} \in\right.$ $W_{y}$ for all $i$. This subspace is not the whole $\mathbf{P}$, as $H^{0}(\partial)(\alpha) \neq 0$.

\section{Projections of syzygies of projective varieties}

Consider $X \subset \mathbf{P}$ a nondegenerate irreducible variety, $x \in \mathbf{P}$ a point, and $Y \subset \mathbf{P} W_{x}^{*}$ the image of $X$ by the projection centered in $x, \mathbf{P} \backslash\{x\} \longrightarrow \mathbf{P} W_{x}^{*}$. Denote by $I_{X}$, and $I_{Y}$ the homogenous ideals, and by $S_{X}$, and $S_{Y}$ the homogeneous coordinate rings of $X$, and $Y$ respectively. We have a natural embedding $S_{Y} \hookrightarrow S_{X}$ which induces, for any integer $p$, an injective map $K_{p, 1}\left(S_{Y}, W_{x}\right) \hookrightarrow K_{p, 1}\left(S_{X}, W_{x}\right)$. A key fact is that $L_{x} \otimes K_{p, 1}\left(S_{Y}, W_{x}\right)$ contains all the projected syzygies, as shown in [Ehb]:

(3.1) Lemma. (Ehbauer) $\operatorname{Im}\left(K_{p+1,1}\left(S_{X}, V\right) \stackrel{\pi_{x}}{\longrightarrow} L_{x} \otimes K_{p, 1}\left(S_{X}, W_{x}\right)\right) \subset L_{x} \otimes$ $K_{p, 1}\left(S_{Y}, W_{x}\right)$, for any $p \geq 1$.

Proof. (cf. Ehb Section 6) Since the image of $X$ in $\mathbf{P}$ is nondegenerate, we have canonical isomorphisms, for any $p \geq 1$,

$$
K_{p+1,1}\left(S_{X}, V\right) \cong K_{p, 2}\left(I_{X}, V\right) \cong \operatorname{Ker}\left(\left(I_{X}\right)_{2} \otimes \bigwedge^{p} V \rightarrow\left(I_{X}\right)_{3} \otimes \bigwedge^{p-1} V\right)
$$

similarly for $Y$. The statement of Lemma reduces then to prove that the projection of an element of $K_{p, 2}\left(I_{X}, V\right)$ belongs to $L_{x} \otimes K_{p-1,2}\left(I_{Y}, W_{x}\right)$.

The see this, we choose homogeneous coordinates $X_{0}, \ldots, X_{r}$ on $\mathbf{P}$, such that $x=\left[\begin{array}{llllll}1 & : & 0 & : & \ldots & :\end{array}\right]$, and all the coordinate points belong to $X$. An element in $K_{p+1,1}\left(S_{X}, V\right)$ can be seen then as a collection of quadrics vanishing on $X$, $\left(Q_{i_{1} \ldots i_{p}}\right)_{0 \leq i_{1}<\ldots<i_{p} \leq r}$, satisfying the equations:

$$
\sum_{k \notin\left\{k_{1}, \ldots, k_{p-1}\right\}}(-1)^{\#\left\{k_{i}<k\right\}} Q_{k_{1} \ldots k \ldots k_{p-1}} X_{k}=0,
$$

for all $0 \leq k_{1}<\ldots<k_{p-1} \leq r$. Projection of syzygies simply means removing all the $Q_{i_{1} \ldots i_{p}}$ 's for $i_{1} \neq 0$, and renaming $\widetilde{Q}_{j_{1} \ldots j_{p-1}}=Q_{0 j_{1} \ldots j_{p-1}}$, for all $1 \leq j_{1}<\ldots<$ $j_{p-1} \leq r$. A simple analysis shows that $\left(\widetilde{Q}_{j_{1} \ldots j_{p-1}}\right)_{1 \leq j_{1}<\ldots<j_{p-1} \leq r}$ actually belongs to $\left(I_{Y}\right)_{2} \otimes \wedge^{p-1} W_{x}$, and the corresponding equations are still preserved. 
In the case of curves, which is the most interesting for our purposes, we can actually prove more.

(3.2) Proposition. If $L$ is a globally generated line bundle over the curve $X$, $x \in X$ is a point, and $W_{x}=H^{0}(X, L-x)$, then, for any $p \geq 1$, the natural map $K_{p, 1}(X, L-x) \rightarrow K_{p, 1}\left(X, L, W_{x}\right)$ is injective, and $\operatorname{Im}\left(K_{p+1,1}(X, L) \stackrel{\pi_{x}}{\longrightarrow} L_{x} \otimes\right.$ $\left.K_{p, 1}\left(X, L, W_{x}\right)\right) \subset L_{x} \otimes K_{p, 1}(X, L-x)$.

Proof. The injectivity follows from (1.1). Let $V=H^{0}(X, L), X^{\prime}$ be the image of $X$ in $\mathbf{P} V^{*}$, and $Y$ be the image of $X^{\prime}$ in $\mathbf{P} W_{x}^{*}$ under the projection centered in $x$. By means of (1.2), we have $K_{p+1,1}\left(S_{X^{\prime}}, V\right) \cong K_{p+1,1}(X, L)$, for all $p$. Using (1.1), we can also show that $K_{p, 1}\left(S_{Y}, W_{x}\right) \cong K_{p, 1}(X, L-x)$. We apply (3.1) to conclude.

(3.3) Corollary. If $L$ is a globally generated line bundle over the curve $X$, and $K_{p+1,1}(X, L) \neq 0$ for an integer $p \geq 1$, then $K_{p, 1}(X, L-x) \neq 0$ for a generic point $x \in X$.

Proof. Observe that, by means of (2.5), any nonzero element in $K_{p+1,1}(X, L) \neq 0$ survives in $K_{p, 1}\left(X, L, W_{x}\right)$ by projecting from a generic point $x \in X$, as the image of $X$ in $\mathbf{P} H^{0}(X, L)^{*}$ is nondegenerate.

\section{Proofs of main results}

Both Theorem 1, and Theorem 3, follow as immediate consequences of a key Lemma which we show next.

(4.1) Lemma. Let $X$ be a curve of genus $g$, and $L_{0}$ be a nonspecial line bundle on $X$ of degree $d \geq g$. If $L_{0}$ satisfies $\left(M_{k}\right)$, and $x_{0}$ is a point of $X$ such that $L=L_{0}+x_{0}$ is globally generated, then $L$ also satisfies $\left(M_{k}\right)$.

Proof. We want to prove that the vanishing of $K_{p, 1}\left(X, L_{0}\right)$, for a positive integer $p$, implies vanishing for $K_{p+1,1}(X, L)$. We use the following notation:

$$
X_{1}:=\left\{x \in X, h^{1}(X, L-x)=0\right\} ;
$$

it is an open set of $X$, and, since it contains the point $x_{0}$, which corresponds to $L_{0}$, it is moreover nonempty. Besides, as under vanishing hypotheses the Koszul complex is actually a complex of vector bundles over the base of deformation, and the locus where it is not exact is closed, we remark that, for any positive integer $p$, the set

$$
X_{p}:=\left\{x \in X_{1}, K_{p, 1}(X, L-x) \neq 0\right\}
$$

is closed in $X_{1}$.

Assume $K_{p+1,1}(X, L) \neq 0$. It follows directly from (3.3) that $K_{p, 1}(X, L-x) \neq 0$ for a generic point $x \in X$, hence $X_{p}$ contains a nonempty open set of $X_{1}$. In this case, it must be the whole $X_{1}$, and, since $x_{0} \in X_{1}$, we see that $K_{p, 1}\left(X, L_{0}\right) \neq 0$.

Proof of Theorem 1. Since for any effective divisor $D, L_{0}+D$ is nonspecial 
and globally generated, as soon as $L_{0}$ itself is nonspecial and globally generated, an inductive argument allows us to reduce to the case of a divisor of degree 1 , which follows from (4.1). For the last part of the statement, observe that $H^{0}\left(X, L-L_{0}\right) \neq 0$, and thus $L$ itself is of type $L_{0}+D$.

(4.2) Corollary. If $L_{0}$ is a nonspecial globally generated line bundle on the curve $X$, which satifies $\left(M_{k}\right)$, then, for any effective divisor $D$, any integer $0 \leq \gamma<$ $\operatorname{deg}(D)$, and $x_{1}, \ldots, x_{\gamma}$ generic points of $X$, the line bundle $L_{0}+D-x_{1}-\ldots-x_{\gamma}$ also satisfies the property $\left(M_{k}\right)$.

Proof. The set of $\gamma$-tuples $\left(x_{1}, \ldots, x_{\gamma}\right)$, for which $L_{0}+D-x_{1}-\ldots-x_{\gamma}$ is nonspecial, globally generated, and satisfies $\left(M_{k}\right)$, is open. Since $D$ is effective and $0 \leq \gamma<$ $\operatorname{deg}(D)$, there exists a set of points of $X,\left\{x_{1}, \ldots, x_{\gamma}\right\}$, such that $D-x_{1}-\ldots-x_{\gamma}$ is effective, hence $L_{0}+D-x_{1}-\ldots-x_{\gamma}$ is nonspecial, globally generated and satisfies the property $\left(M_{k}\right)$. Therefore, the above-mentioned open set is nonempty.

Proof of Theorem 3. If $x$ is a point of $X$, and $L_{0}=K_{X}+x$, then $L_{0}$ is nonspecial, and $H^{0}\left(X, K_{X}\right) \cong H^{0}\left(X, L_{0}\right)$. In particular, for any $p, K_{p, 1}\left(X, K_{X}\right) \cong K_{p, 1}\left(X, L_{0}\right)$, and thus $L_{0}$ satisfies $\left(M_{k}\right)$. Then we apply (4.1) for $L_{0}$.

\section{Syzygies of plane curves}

One of the main results of $[\mathrm{LO}]$ shows that Green's canonical conjecture is true for a plane curve. The main idea of his proof was to relate the Koszul cohomology of the projective plane, by means of a long cohomology sequence, to the Koszul cohomology of the curve. In this Section, we use a similar strategy to prove that the gonality conjecture also holds for plane curves.

We begin by pointing out the following useful fact:

(5.1) Lemma. For any integers $k \geq 2$, and $p \geq 1, K_{p, 1}\left(\mathbf{P}^{2}, \mathcal{O}_{\mathbf{P}^{2}}(k)\right)=0$ if and only if $p \geq N(k):=h^{0} \mathcal{O}_{\mathbf{P}^{2}}(k)-k$.

Proof. Let $r=h^{0} \mathcal{O}_{\mathbf{P}^{2}}(k)-1$. Green's duality (Gr1, Corollary 2.c.10) in this case translates into

$$
K_{p, 1}\left(\mathbf{P}^{2}, \mathcal{O}_{\mathbf{P}^{2}}(k)\right) \cong K_{r-p-2,2}\left(\mathbf{P}^{2}, \mathcal{O}_{\mathbf{P}^{2}}(-3), \mathcal{O}_{\mathbf{P}^{2}}(k)\right)^{*}
$$

For $p \geq N(k)$, we have $r-p-2 \leq-3+k$, so we can apply Theorem 2.2 of [Gr2], or Theorem 4.1 of [Gr3] to get the vanishing of $K_{p, 1}\left(\mathbf{P}^{2}, \mathcal{O}_{\mathbf{P}^{2}}(k)\right)$. The fact that this bound is sharp easily follows from [GL1], by decomposing $\mathcal{O}_{\mathbf{P}^{2}}(k)=\mathcal{O}_{\mathbf{P}^{2}}(k-1) \otimes$ $\mathcal{O}_{\mathbf{P}^{2}}(1)$.

Let $X \subset \mathbf{P}^{2}$ be a smooth plane curve of degree $k+1=d \geq 3$. We prove:

(5.2) Proposition. The pair $\left(X, \mathcal{O}_{X}(k)\right)$ satisfies the property $\left(M_{k-1}\right)$. 
Proof. We denote $V=H^{0} \mathcal{O}_{\mathbf{P}^{2}}(k)=H^{0} \mathcal{O}_{X}(k)$, and consider the exact sequence of graded $S V$-modules:

$$
0 \longrightarrow \bigoplus_{q \geq 0} H^{0} \mathcal{O}_{\mathbf{P}^{2}}(-d+q k) \longrightarrow \bigoplus_{q \geq 0} H^{0} \mathcal{O}_{\mathbf{P}^{2}}(q k) \longrightarrow \bigoplus_{q \geq 0} H^{0} \mathcal{O}_{X}(q k) \longrightarrow 0
$$

together with its associated long cohomology sequence:

$$
\begin{gathered}
\ldots \rightarrow K_{p, 1}\left(\mathbf{P}^{2}, \mathcal{O}_{\mathbf{P}^{2}}(-d), \mathcal{O}_{\mathbf{P}^{2}}(k)\right) \rightarrow K_{p, 1}\left(\mathbf{P}^{2}, \mathcal{O}_{\mathbf{P}^{2}}(k)\right) \rightarrow K_{p, 1}\left(X, \mathcal{O}_{X}(k)\right) \rightarrow \\
\rightarrow K_{p-1,2}\left(\mathbf{P}^{2}, \mathcal{O}_{\mathbf{P}^{2}}(-d), \mathcal{O}_{\mathbf{P}^{2}}(k)\right) \rightarrow \ldots
\end{gathered}
$$

By means of Green's Vanishing Theorem (cf. [Gr1], Theorem 3.a.1), we see that $K_{p, 1}\left(\mathbf{P}^{2}, \mathcal{O}_{\mathbf{P}^{2}}(-d), \mathcal{O}_{\mathbf{P}^{2}}(k)\right)=0$ for all $p \geq 0$, and $K_{p-1,2}\left(\mathbf{P}^{2}, \mathcal{O}_{\mathbf{P}^{2}}(-d), \mathcal{O}_{\mathbf{P}^{2}}(k)\right)=$ 0 for all $p \geq N(k)$. Therefore, for all $p \geq N(k)$, we have $K_{p, 1}\left(\mathbf{P}^{2}, \mathcal{O}_{\mathbf{P}^{2}}(k)\right) \cong$ $K_{p, 1}\left(X, \mathcal{O}_{X}(k)\right)$, and the Lemma follows as a direct consequence of (5.1).

In this special case, Corollary 2 applied to $\mathcal{O}_{X}(k)$ translates into the following (the first part of the statement has been known for a long time: see, for example, ACGH p.56)

(5.3) Corollary. The curve $X$ is $k$-gonal, a pencil of minimal degree being obtained by projecting from a point of the curve, and the gonality conjecture is valid for $X$.

The property $\left(M_{k-1}\right)$ is fulfilled for any line bundle $\mathcal{O}_{X}(n)$, with $n \geq k$, and it fails for $\mathcal{O}_{X}(k-2)=K_{X}$. We can inquire about $\mathcal{O}_{X}(k-1)$, and address the question of whether it satisfies $\left(M_{k-1}\right)$ or not. The answer is NO: since the canonical maps $K_{p, 1}\left(\mathbf{P}^{2}, \mathcal{O}_{\mathbf{P}^{2}}(k-1)\right) \rightarrow K_{p, 1}\left(X, \mathcal{O}_{X}(k-1)\right)$ are injective, for all positive $p$ (Green's vanishing and the long exact sequence, as above), the second part of (5.1) applied for $(k-1)$ shows that the property $\left(M_{k-1}\right)$ fails for $\left(X, \mathcal{O}_{X}(k-1)\right)$. It is natural then to ask about intermediate line bundles (see also (6.4) below):

(5.4) Problem. Find the least $\gamma$ such that, for any colinear points $x_{1}, \ldots, x_{\gamma} \in X$, the property $\left(M_{k-1}\right)$ fails for the line bundle $\mathcal{O}_{X}(k) \otimes \mathcal{O}_{X}\left(-x_{1}-\ldots-x_{\gamma}\right)$.

\section{Syzygies of curves on a Hirzebruch surface}

Let $\Sigma_{e}$ be the Hirzebruch surface of invariant $e$, and denote by $C_{0}$ the minimal section of $\Sigma_{e}$, and by $f$ a fiber of the ruling. For any integers $a$, and $b$, we consider the line bundle on $\Sigma_{e}, H_{a, b}=(a-1) C_{0}+(b-1) f$, to whom we attach the integer $N(a, b):=$ $h^{0} \mathcal{O}_{\Sigma_{e}}\left(H_{a, b}\right)-a$. We choose two integers $k \geq 2$, and $m \geq \max \{k e, k+e\}$, and a curve $X \in\left|H_{k+1, m+1}\right|$; its genus is computed by the formula $g=(k-1)(m-1-k e / 2)$.

We will show that the pair $\left(X,\left.H_{k, m}\right|_{X}\right)$ satisfies the property $\left(M_{k-1}\right)$. For this, we need two preliminary Lemmas.

(6.1) Lemma. We have a natural isomorphism $K_{p, 1}\left(\Sigma_{e}, H_{k, m}\right) \cong K_{p, 1}\left(X,\left.H_{k, m}\right|_{X}\right)$, for any $p \geq N(k, m)$. 
Proof. We denote by $V=H^{0} \mathcal{O}_{\Sigma_{e}}\left(H_{k, m}\right)=H^{0}\left(X,\left.\mathcal{O}_{\Sigma_{e}}\left(H_{k, m}\right)\right|_{X}\right)$, and consider the graded $S V$-modules: $B^{\prime}=\bigoplus_{q \geq 0} H^{0} \mathcal{O}_{\Sigma_{e}}\left(-X+q H_{k, m}\right), B=\bigoplus_{q \geq 0} H^{0} \mathcal{O}_{\Sigma_{e}}\left(q H_{k, m}\right)$, and $A=B / B^{\prime}$. We have then a long exact sequence:

$$
\begin{gathered}
\ldots \rightarrow K_{p, 1}\left(\Sigma_{e},-X, H_{k, m}\right) \rightarrow K_{p, 1}\left(\Sigma_{e}, H_{k, m}\right) \rightarrow K_{p, 1}(A, V) \rightarrow \\
\rightarrow K_{p-1,2}\left(\Sigma_{e},-X, H_{k, m}\right) \rightarrow \ldots
\end{gathered}
$$

Since $A_{0} \cong \mathbf{C}, A_{1} \cong H^{0}\left(X,\left.\mathcal{O}_{\Sigma_{e}}\left(H_{k, m}\right)\right|_{X}\right)$, and $A_{2} \subset H^{0}\left(X,\left.\mathcal{O}_{\Sigma_{e}}\left(2 H_{k, m}\right)\right|_{X}\right),(1.1)$ shows that $K_{p, 1}(A, V) \cong K_{p, 1}\left(X,\left.\mathcal{O}_{\Sigma_{e}}\left(H_{k, m}\right)\right|_{X}\right)$.

We apply again Green's Vanishing Theorem ([Gr1], Theorem 3.a.1), which implies $K_{p, 1}\left(\Sigma_{e},-X, H_{k, m}\right)=0$ as soon as $p \geq 0$, and $K_{p-1,2}\left(\Sigma_{e},-X, H_{k, m}\right)=0$ for all $p \geq h^{0} \mathcal{O}_{\Sigma_{e}}\left(H_{k-1, m-1}\right)+1$. Since $N(k, m) \geq h^{0} \mathcal{O}_{\Sigma_{e}}\left(H_{k-1, m-1}\right)+1$. we get isomorphisms $K_{p, 1}\left(\Sigma_{e}, H_{k, m}\right) \cong K_{p, 1}\left(X,\left.H_{k, m}\right|_{X}\right)$, for all $p \geq N(k, m)$, as stated.

(6.2) Lemma. For all $p \geq N(k, m)$, we have $K_{p, 1}\left(\Sigma_{e}, H_{k, m}\right)=0$.

Proof. It suffices to prove vanishing for $p=N(k, m)$ only, so we stick to this case.

We consider $Y$ a curve in the linear system $\left|H_{k, m}\right|$ on $\Sigma_{e}$. Then $h^{0}\left(Y,\left.H_{k, m}\right|_{Y}\right)=$ $h^{0}\left(\Sigma_{e}, H_{k, m}\right)-1$, and the surjection $V \rightarrow H^{0}\left(Y,\left.H_{k, m}\right|_{Y}\right)$ corresponds to the hyperplane of $\mathbf{P} V^{*}$ which cuts out the curve $Y$ on the image of $\Sigma_{e}$. Since we work on a rational surface, (1.3) applies, and we get an isomorphism $K_{N(k, m), 1}\left(\Sigma_{e}, H_{k, m}\right) \cong$ $K_{N(k, m), 1}\left(Y,\left.H_{k, m}\right|_{Y}\right)$.

We prove the Lemma by induction on $k$; the first step is $k=2$. In this case, $N(2, m)=h^{0}\left(Y,\left.H_{2, m}\right|_{Y}\right)-1$; by the general theory of syzygies (apply, for example, Theorem 3.c.1 (1) of [Gr1]), we know that $K_{h^{0}\left(Y,\left.H_{2, m}\right|_{Y}\right)-1,1}\left(Y,\left.H_{2, m}\right|_{Y}\right)=0$.

The induction step: for $k \geq 3$ we know $K_{N(k-1, m-1), 1}\left(\Sigma_{e}, H_{k-1, m-1}\right)=0$, and we wish to prove that $K_{N(k, m), 1}\left(\Sigma_{e}, H_{k, m}\right)=0$, i.e. $K_{N(k, m), 1}\left(Y,\left.H_{k, m}\right|_{Y}\right)=0$.

Lemma (6.1) applied for $Y$ yields an isomorphism $K_{N(k-1, m-1), 1}\left(Y,\left.H_{k-1, m-1}\right|_{Y}\right) \cong$ $K_{N(k-1, m-1), 1}\left(\Sigma_{e}, H_{k-1, m-1}\right)$, which shows that $K_{N(k-1, m-1), 1}\left(Y,\left.H_{k-1, m-1}\right|_{Y}\right)=0$. The induction step is completed by means of the following facts:

$$
\begin{gathered}
H_{k-1, m-1} . Y \geq 2 g(Y)+1, \\
\left|\left(H_{k, m}-H_{k-1, m-1}\right)\right|_{Y} \mid \neq \emptyset,
\end{gathered}
$$

so there exists $D \in\left|\left(H_{k, m}-H_{k-1, m-1}\right)\right|_{Y} \mid$,

$$
N(k, m)-N(k-1, m-1)=\left(H_{k, m}-H_{k-1, m-1}\right) \cdot H_{k, m}=\operatorname{deg}(D),
$$

which altogether permit us to apply Theorem 1 for $Y$, and the line bundles $L_{0}=$ $\left.H_{k-1, m-1}\right|_{Y}, L=\left.H_{k, m}\right|_{Y}=L_{0}+D$.

The obvious inequality $H_{k, m} . X \geq 2 g+1$, together with the existence of a $g_{k}^{1}$ given by the ruling, show that the gonality conjecture is verified for $X$ (the fact that $X$ is $k$-gonal was previously proved in Ma1, by using completely different methods):

(6.3) Theorem. Let $e \geq 0, k \geq 2$, and $m \geq \max \{k e, k+e\}$ be three integers, and $X \equiv k C_{0}+m f$ be a curve on the Hirzebruch surface $\Sigma_{e}$. Then $X$ is $k$-gonal, 
a pencil of minimal degree being given by the ruling, and for any $L \in \operatorname{Pic}(X)$ with $h^{0}\left(X,\left.L \otimes \mathcal{O}_{\Sigma_{e}}\left(-H_{k, m}\right)\right|_{X}\right) \neq 0$ the property $\left(M_{k-1}\right)$ holds for $(X, L)$.

In the particular case $e=1, m=k+1$, the curve $X$ is the strict transform of a plane curve $X_{0}$ of degree $k+1$, which passes through the center $x_{0}$ of the blowup. Via the natural isomorphism between $X$ and $X_{0}$, the line bundle $\left.H_{k, k+1}\right|_{X}$ corresponds to $\mathcal{O}_{X_{0}}(k) \otimes \mathcal{O}_{X_{0}}\left(-x_{0}\right)$. This shows that (6.3) does a bit better than $(5.2)$.

\section{The gonality conjecture for generic nodal curves on $\mathbf{P}^{1} \times \mathbf{P}^{1}$}

The aim of this Section is to prove that the gonality conjecture is valid for a nodal curve on $\mathbf{P}^{1} \times \mathbf{P}^{1}$ whose singular points are in general position, fact which makes the proof of Theorem 4 be straightforward; this idea was inspired by the work [Sch2. We freely use the notation of the previous Sections.

We consider three integers $k \geq 3, m \geq k, 0 \leq \gamma \leq k-2, Y \in\left|H_{k, m}\right|$ a smooth curve of genus $(k-2)(m-2)$ on $\mathbf{P}^{1} \times \mathbf{P}^{1}, L_{0}=\left.H_{k-1, m-1}\right|_{Y}$, and $L=\left.H_{k, m}\right|_{Y}$. Then (6.3) applies for $Y$, hence the pair $\left(Y, L_{0}\right)$ satisfies $\left(M_{k-2}\right)$. In this case, for a set of general points $\left\{x_{1}, \ldots, x_{\gamma}\right\} \subset Y$ the line bundle $L-x_{1}-\ldots-x_{\gamma}$ satisfies the property $\left(M_{k-2}\right)$ (cf. (4.2)). Without loss of generality, this set can be chosen such that the points $\left\{x_{1}, \ldots, x_{\gamma}\right\}$ are in general position in $\mathbf{P}^{1} \times \mathbf{P}^{1}$, in the sense that any two of them are not colinear. We consider furthermore $\Sigma \stackrel{\sigma}{\rightarrow} \mathbf{P}^{1} \times \mathbf{P}^{1}$ the blowup of the points $\left\{x_{1}, \ldots, x_{\gamma}\right\}, E$ the exceptional divisor, $H=\sigma^{*}(k-1, m-1)-E$, and $\tilde{Y} \in|H|$ the strict transform of $Y$.

Proposition 1 of Sch2 ensures the existence of a smooth connected curve in the linear system $\left|\sigma^{*}(k, m)-2 E\right|$; let us denote it by $X$. The curve $X$ is $k$-gonal (see below), its genus equals $g=(k-1)(m-1)-\gamma$, and its projection on $\mathbf{P}^{1} \times \mathbf{P}^{1}$ is an irreducible curve of type $(k, m)$, with assigned ordinary nodes at $\left\{x_{1}, \ldots, x_{\gamma}\right\}$.

(7.1) Proposition. The pair $\left(X,\left.H\right|_{X}\right)$ satisfies the property $\left(M_{k-1}\right)$.

Proof. It is easy to see that $h^{i} \mathcal{O}_{\Sigma}(H-X)=0$ for all $i$. Then we denote $V=$ $H^{0} \mathcal{O}_{\Sigma}(H) \cong H^{0}\left(X,\left.\mathcal{O}_{\Sigma}(H)\right|_{X}\right)$, and compute $\operatorname{dim}(V)-k=k(m-1)-\gamma$. In a similar way as in the Proof of (6.1), we can check that $\operatorname{dim}(V)-k \geq h^{0} \mathcal{O}_{\Sigma}(2 H-X)+1$, hence $K_{p, 1}(\Sigma, H) \cong K_{p, 1}\left(X,\left.H\right|_{X}\right)$ for all $p \geq \operatorname{dim}(V)-k$. Moreover, (1.3) applied to this case shows that $K_{p, 1}(\Sigma, H) \cong K_{p, 1}\left(\tilde{Y},\left.H\right|_{\tilde{Y}}\right)$. Now, the restriction of $\sigma$ to $\tilde{Y}$ gives a natural isomorphism to $Y$; the line bundle $\left.H\right|_{\widetilde{Y}}$ on $\tilde{Y}$ corresponds to the line bundle $L-x_{1}-\ldots-x_{\gamma}$ on $Y$. Besides, Riemann-Roch implies $h^{0}\left(Y, L-x_{1}-\ldots-x_{\gamma}\right)-(k-1)=$ $\operatorname{dim}(V)-k$. Since $L-x_{1}-\ldots-x_{\gamma}$ satisfies $\left(M_{k-2}\right)$, i.e. $K_{p, 1}\left(Y, L-x_{1}-\ldots-x_{\gamma}\right)=0$ for $p \geq h^{0}\left(Y, L-x_{1}-\ldots-x_{\gamma}\right)-(k-1)$, we are done.

Consequently, as $H . X \geq 2 g+1$, and $X$ carries a $g_{k}^{1}$, the gonality conjecture is verified for $X$, and thus Theorem 4 is true. 


\section{Application: Curves on Hirzebruch surfaces satisfy Green's canonical conjecture}

In his paper Gr1, Green conjectured that, given $X$ a curve of genus $g$, and denoting by $c$ its Clifford index (we refer to [Ma2 for a precise definition) all $K_{p, 1}\left(X, K_{X}\right)$ vanish for $p \geq g-c-1$. In other words, the line bundle $K_{X}$ satisfies the property $\left(M_{c}\right)$.

The aim of this Section is to verify this conjecture for curves on Hirzebruch surfaces (we use the same notation as in Section 6).

(8.1) Theorem. Let $\Sigma_{e}$ be the Hirzebruch surface of invariant $e$, and $X$ be a curve on $\Sigma_{e}$, numerically equvalent to $k C_{0}+m f$, with $k \geq 3$ and $m \geq \max \{k e+$ $1, k+1, k+2 e\}$. Then the Clifford dimension of $X$ equals one, and Green's canonical conjecture is valid for $X$.

Proof. We start with the exact sequence, for any $q$ :

$$
0 \longrightarrow \mathcal{O}_{\Sigma_{e}}(q H-X) \longrightarrow \mathcal{O}_{\Sigma_{e}}(q H) \longrightarrow K_{X} \longrightarrow 0
$$

where $H=K_{\Sigma_{e}}+X$. Observe that $H^{0} \mathcal{O}_{\Sigma_{e}}(H-X)=H^{1} \mathcal{O}_{\Sigma_{e}}(H-X)=0$, and thus $H^{0} \mathcal{O}_{\Sigma_{e}}(H) \cong H^{0}\left(X, K_{X}\right)$. Obviously, their common dimension equals the genus of $X$. We get next a long exact sequence (see (1.1), and [Gr1] 1.d.4):

$$
\ldots \rightarrow K_{p, 1}\left(\Sigma_{e},-X, H\right) \rightarrow K_{p, 1}\left(\Sigma_{e}, H\right) \rightarrow K_{p, 1}\left(X, K_{X}\right) \rightarrow K_{p-1,2}\left(\Sigma_{e},-X, H\right) \rightarrow \ldots
$$

By means of Green's vanishing theorem (Gr1 3.a.1), $K_{p-1,2}\left(\Sigma_{e},-X, H\right)=0$ for $p \geq$ $h^{0} \mathcal{O}_{\Sigma_{e}}(2 H-X)+1$. From the inequality $m \geq \max \{k+2 e, k e+1\}$, we see that Lemma (6.2) applies, and thus $K_{p, 1}\left(\Sigma_{e}, H\right)=0$ for all $p \geq h^{0} \mathcal{O}_{\Sigma_{e}}(H)-(k-1)=g-k+1$. To conclude the proof, we remark that, on the one hand, $h^{0} \mathcal{O}_{\Sigma_{e}}(2 H-X) \leq g-k$, which implies the vanishing of $K_{p, 1}\left(X, K_{X}\right)$ for all $p \geq g-k+1$, and, on the other hand, $X$ naturally carries a $g_{k}^{1}$.

(8.2) Remark. The role of the vanishing result (6.2) is similar to the one of Green's vanishing [Gr2] 2.2 for the case of plane curves, studied in [LO].

(8.3) Remark. By following a similar strategy, one can easily prove that Green's canonical conjecture is true for certain nodal curves on $\mathbf{P}^{1} \times \mathbf{P}^{1}$. To do that, we simply apply (7.1), and addapt the proof of (8.1) to the new framework. This also provides a new proof of the main result of [Sch1], which says that Green's canonical conjecture is true for generic curves whose genera are sufficiently large compared to the gonality.

Acknowledgements. I would like to express my gratitude to F.-O. Schreyer for guiding my first steps in this topic, as well as for the many enlightening discussions we had, and suggestions which I benefited from. I am also grateful to C. Voisin for valuable comments on an early version of the manuscript.

This work has been carried out while I was visiting the University of Bayreuth, the Abdus Salam ICTP (Trieste), and the Fourier Institute (Grenoble), to whom I address my thanks for hospitality. 


\section{References}

[ACGH] E. Arbarello, M. Cornalba, P. A. Griffiths and J. Harris, Geometry of algebraic curves, Volume I. Grundlehren der mathematischen Wissenschaften, 267. Springer-Verlag. XVI, (1985).

[Ehb] S. Ehbauer, Syzygies of points in projective space and applications, Orecchia, Ferruccio (ed.) et al., Zero-dimensional schemes. Proceedings of the international conference held in Ravello, Italy, June 8-13, 1992. Berlin: de Gruyter. (1994) $145-170$

[EL] L. Ein and R. Lazarsfeld, Syzygies and Koszul cohomology of smooth projective varieties of arbitrary dimension, Invent. Math. 111 (1993) 51-67

[ELMS] D. Eisenbud, H. Lange, G. Martens, F.-O. Schreyer, The Clifford dimension of a projective curve, Compositio Math. 72 (1989), no. 2, 173-204

[F] W. Fulton, Hurwitz schemes and irreducibility of moduli of algebraic curves, Ann. Math. 90 (1969) 541-575

[Gr1] M. Green, Koszul cohomology and the geometry of projective varieties, J. Diff. Geom. 19 (1984) 125-171

[Gr2] M. Green, Koszul cohomology and the geometry of projective varieties II, J. Diff. Geom. 20 (1984) 279-289

[Gr3] M. Green, Koszul cohomology and geometry, M. Cornalba (ed.) et al., Proceedings of the first college on Riemann surfaces held in Trieste, Italy, November 9-December 18, 1987. Teaneck, NJ: World Scientific Publishing Co. (1989) 177200

[GL1] M. Green and R. Lazarsfeld, The nonvanishing of certain Koszul cohomology groups, J. Diff. Geom. 19 (1984) 168-170 (Appendix to [Gr1])

[GL2] M. Green and R. Lazarsfeld, On the projective normality of complete linear series on an algebraic curve, Invent. Math. 83 (1986) 73-90

[La1] R. Lazarsfeld, A sampling of vector bundle techniques in the study of linear series, M. Cornalba (ed.) et al., Proceedings of the first college on Riemann surfaces held in Trieste, Italy, November 9-December 18, 1987. Teaneck, NJ: World Scientific Publishing Co. (1989) 500-559

[La2] R. Lazarsfeld, Linear series on algebraic varieties, Proc. Int. Congr. Math., Kyoto/Japan 1990, Vol. I (1991) 715-723

[Lo] F. Loose, On the graded Betti numbers of plane algebraic curves, Manuscripta Math. 64 (1989) 503-514

[Ma1] G. Martens, The gonality of curves on a Hirzebruch surface, Arch. Math. 64 (1996) 349-352

[Ma2] G. Martens, Über den Clifford-Index algebraischer Kurven, J. Reine Angew. Math. 320 (1982) 68-85 
[Sch1] F.-O. Schreyer, Syzygies of canonical curves and special linear series, Math. Ann. 275 (1986) 105-137

[Sch2] F.-O. Schreyer, Green's conjecture for general p-gonal curves of large genus, Algebraic curves and projective geometry, Trento, 1988, Lecture Notes in Math., 1389, Springer, Berlin-New York (1989) 254-260.

[Sch3] F.-O. Schreyer, A standard basis approach to syzygies of canonical curves, J. Reine Angew. Math. 421 (1991), 83-123.

[V1] C. Voisin, Courbes tétragonales et cohomologie de Koszul, J. Reine Angew. Math. 387 (1988), 111-121.

[V2] C. Voisin, Green's canonical syzygy conjecture for curves of even genus lying on K3 surfaces, Preprint 2001. 